\title{
Autosomal recessive distal renal tubular acidosis
}

INSERM

\section{Source}

INSERM. (1999). Orphanet: an online rare disease and orphan drug data base. Autosomal recessive distal renal tubular acidosis. ORPHA:402041

Autosomal recessive distal renal tubular acidosis (AR dRTA) is an inherited form of distal renal tubular acidosis (dRTA; see this term) characterized by hypokalemic hyperchloremic metabolic acidosis. Deafness often occurs either early or later on in life but may be absent or never be diagnosed. 